\title{
Comparative evaluation of two commonly administered regimens of mifepristone and misoprostol for first trimester abortion
}

\author{
L. Thulasi Devi ${ }^{1}$, Ravi Nimonkar ${ }^{2 *}$
}

\begin{abstract}
${ }^{1}$ Department of Obstetrics and Gynecology, ${ }^{2}$ Department of Preventive, Social Medicine and Epidemiology, OC, SHO, Command Hospital Eastern Command, Kolkata, West Bengal, India
\end{abstract}

Received: 28 April 2018

Accepted: 25 May 2018

\author{
*Correspondence: \\ Dr. Ravi Nimonkar, \\ E-mail: ravinimonkar1175@gmail.com
}

Copyright: (c) the author(s), publisher and licensee Medip Academy. This is an open-access article distributed under the terms of the Creative Commons Attribution Non-Commercial License, which permits unrestricted non-commercial use, distribution, and reproduction in any medium, provided the original work is properly cited.

\begin{abstract}
Background: The objective of this study was to compare and evaluate the efficacy of two commonly administered regimens as per existing guidelines of Ministry of Health and Family Welfare for Outpatient MTP services. This study is aimed at evaluation of subjective and objective stastical benefits and side effects in performance of first trimester abortion on OPD basis in popularly used drugs as advised by MOHFW by different routes of administration. The drugs used were Tab Mifepristone (RU - 486) and Tab Misoprostol.

Methods: This prospective randomized study was conducted in Out Patient Department of Obstetrics and Gynaecology in a tertiary care hospital over a period of 1 year after due clearance was obtained from Ethical Committee. This was prospective study involving 400 ladies reporting for Outpatient MTP services within the given time period as per existing guidelines at a tertiary care hospital. Patients were assessed at the end of 7, 15and 56 days, the mean age of the patients was $24.5 \pm 0.5$ and $33 \pm 1$ years and treatment duration was an average of 3 days with follow up for 15 days post administration. Few cases of failure required a follow up of approximately 56 days. All patients were on follow up for a period of 3 months for determination of menstrual irregularities and contraception management.

Results: Between the 2 groups, in the sublingual Misoprostol group 100\% aborted successfully at the end of 56 days. Whereas in the vaginal Misoprostol group $99 \%$ aborted successfully at the end of 56 days, only two patients requiring MVA as an OPD procedure.

Conclusions: Patients were assessed at the end of 7, 15 and 56 days and between both the groups; Mifepristone with administration of misoprostol sublingually showed better success rate in completion of procedure, quicker action with better patient satisfaction and acceptance compared to conventional administration of Mifepristone and vaginal administration of misoprostol. Misoprostol administered sublingually under medical supervision is a superior, faster abortificient and has lesser incidence of Retained Products of Conception (RPOC) or need for Suction \& Evacuation (S\&E) as compared to vaginal route. Side effects observed need more evaluation with larger sample size to be statistically significant.
\end{abstract}

Keywords: First trimester abortion, Mifepristone, Misoprostol

\section{INTRODUCTION}

Unsafe abortions are the major cause of complications, morbidity and mortality among women all over the world. Abortions are illegal till date in many countries and non availability of safe, self administered and trusted abortion choices force many women to resort to clandestine induced abortions often leading to death. This limits freedom of sex, sexuality and contraceptive choices among women and is denied right to safe and clean 
clinics with privacy. Maternal deaths due to unsafe abortion practices are approximately $13 \%$ of MMR. Surgical abortion by means of MVA and S\&E remain method of choice among clinicians and patients alike for MTP upto 63 days of gestational age. ${ }^{1}$ In the last several decades many medical, surgical and combined methods have been researched and studied for safety and efficacy for MTP. Various clinical protocols currently advocate use of Mifepristone and Misoprostol for first and second trimester abortions, incomplete and missed abortions, fetal demise, induction and augmentation of labor in different dosages as specified by various regulatory bodies with specified indications and contraindications with due caution. ${ }^{2}$

Here we compare two common routes of administration of Misoprostol that is vaginally and sublingually.

\section{METHODS}

This prospective observational study was conducted between January 2017 to December 2017 and a total of 200 women with early pregnancy who requested first trimester abortion and met the inclusion criteria were randomly assigned into Group A and B. Primary outcome was determined by clinical examination and TVS for complete termination of pregnancy and efficacy recorded. Need of a subsequent dose of Misoprostol, need of surgical interference or procedure and occurrence of complications both major and minor. Secondary outcome were satisfaction levels, acceptability, amount of bleeding, pain and discomfort, ease of administration, need for a visit to the centre out of schedule and willingness to recommend to other patients as a safe method of MTP.

\section{Inclusion criteria}

Age $>18$ years, haemoglobin $>10$ gm $\%$, well informed written consent for termination and consent for medical and follow up surgical procedure, confirmed single Intrauterine gestation and exclusion of extrauterine sac, Mean Sac Diameter <35 mm, Crown Rump Length <2.5 $\mathrm{cm}$, Gestational Age <63 days, Exclusion of drug Hypersensitivity, Access to health facility for OPD and emergency services.

\section{Exclusion criteria}

Age $<18$ years, Anaemia, Lack of consent, inability to demonstrate intrauterine Gestational Sac, Multiple Gestation, uterine malformation, Mean Sac Diameter > $35 \mathrm{~mm}$, Crown Rump Length $>2.5 \mathrm{~cm}$, Gestational Age > 64 days, Drug hypersensitivity, Lack of access to health care facilities, known Cardiovascular, Hepatic or Renal Pathology, Bleeding disorders or Coagulopathies, history of Classical caesarean section or myomectomy, refusal to take abortificient on OPD basis or refusal for follow up surgical procedure if required.

\section{Study methodology}

The present study aimed to study the effectiveness of orally administered Mifepristone 200mg on Day1 and Misoprostol $600 \mu \mathrm{g}$ self administered sublingually after 48 hours (Group A); and compare with 200mg on Day1 and Misoprostol $600 \mu \mathrm{g}$ self administered vaginally after 48 hours (Group B). A total of 200 consecutive cases of early intrauterine pregnancy of gestational age less than 63 days reporting to GOPD, who desired MTP services above the age of 18 years fulfilling the above criteria were selected for this study. The subjects of mixed ethnicity were randomly grouped into Group A and Group B of 200 each with similar clinical presentations. Tab Mifepristone (RU-486) is commonly used progesterone antagonist which acts on receptors of endometrium and trophoblast, allowing prostaglandins to stimulate uterine contractions and causing the conceptus to detach from uterine wall. Various vascular changes are produced which decrease placental viability, act on corpus luteum of pregnancy, decrease glandular secretory activity, accelerate degenerative changes, and increase stromal but not glandular mitotic activity. Misoprostol which is a commonly used recommended Prostaglandin (PGE1) as an abortificient. Misoprostol is a synthetic analogue of alprostadil. It causes myometrial contractions by its interaction with receptors which results in change in Calcium concentration thereby softening the cervix and causing smooth muscle contractions in the corpus uteri thereby causing expulsion of the products.

Initial evaluation was carried out by detailed history and clinical examination to rule out any systemic diseases, coagulation and liver disorders, diabetes mellitus and thyroid dysfunction. Before starting drug therapy an initial haemogram, Blood group \& Trans Vaginal Ultrasound (TVS) in all cases to confirm gestational age and location of sac was done. Well written informed consent was obtained and guidelines laid down by MTP Act were diligently followed. The side effects and complications in both the arms were noted and compared for evaluation of acceptability and efficacy of both arms.

\section{Drug schedule}

Group A was administered Mifepristone $200 \mathrm{mg}$ orally on D1 followed by Misoprostol $600 \mu \mathrm{g}$ sublingually after 36 to 48 hours. Group B was administered Mifepristone $200 \mathrm{mg}$ orally on D1 followed by Misoprostol $600 \mu \mathrm{g}$ orally after 36 to 48 hours. Both dosages were self administered after diligent explanation by physician and

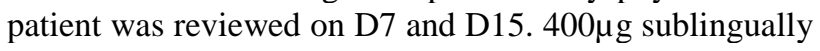
was readministered if on clinical and ultrasound (TVS) reassessment evidence of RPOC was seen. All patients were reviewed on D5 of next menstrual cycle for completion of procedure, contraception management and further surgical intervention if required. They were reviewed earlier in case of complications, complaints or exaggeration of common side effects. Analgesics were prescribed to all patients in anticipation of pain. If the 
gestational sac was intact and cardiac activity was demonstrable on D7 on TVS patient received gynaecological examination and surgical abortion was done.

\section{RESULTS}

Table 1: Period of gestation in weeks in each arm.

\begin{tabular}{|lll|}
\hline $\begin{array}{l}\text { Period of } \\
\text { amenorrhoea } \\
\text { (in Weeks) }\end{array}$ & $\begin{array}{l}\text { No of patients } \\
\text { Group A } \\
(\mathbf{N}=200)\end{array}$ & $\begin{array}{l}\text { No of patients } \\
\text { Group B } \\
(\mathbf{N}=200)\end{array}$ \\
\hline $3+^{6}$ & 2 & 2 \\
\hline $4+^{6}$ & 10 & 8 \\
\hline $5+^{6}$ & 16 & 14 \\
\hline $6+^{6}$ & 18 & 16 \\
\hline $7+^{6}$ & 48 & 44 \\
\hline $8+^{6}$ & 54 & 62 \\
\hline 9 & 52 & 54 \\
\hline
\end{tabular}

It can be seen from the distribution of this chart that maximum number of patients seeking MTP services were at approximate $8+{ }^{6}$ weeks period of gestation.

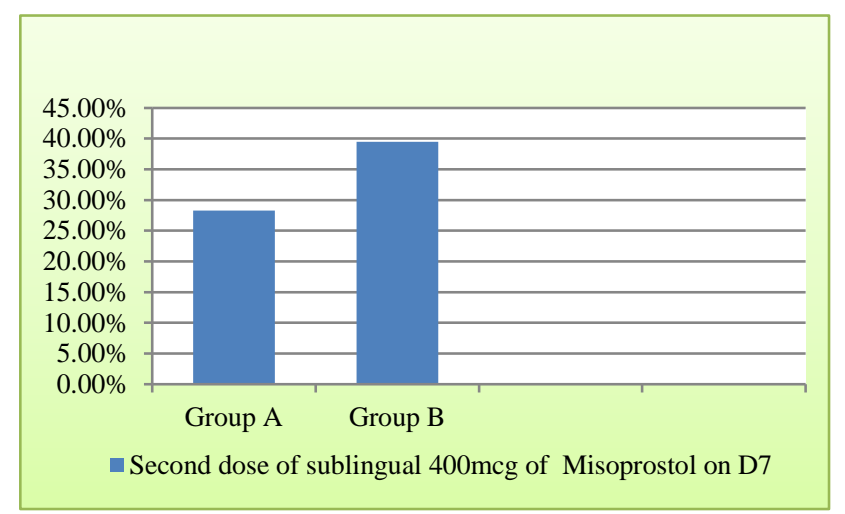

Figure 1: Administration of second dose of $400 \mu \mathrm{g}$ of sublingual misoprostol.

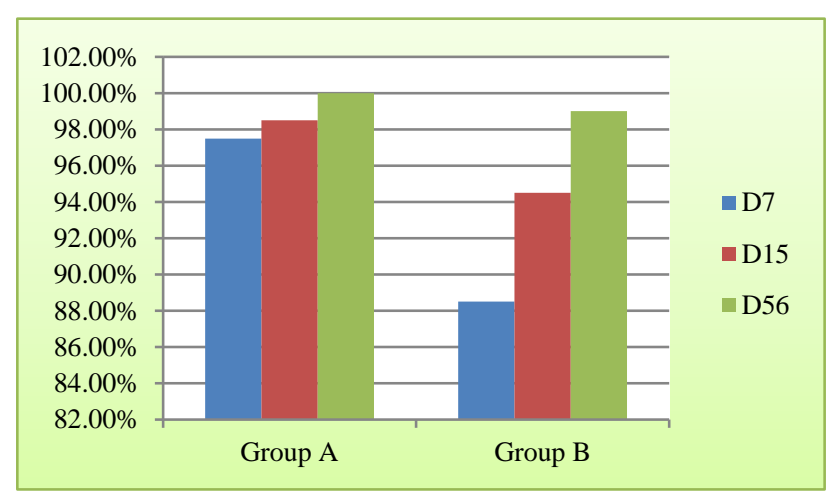

Figure 2: Completeness of abortion at the end of D7, D15 \& D56 as assessed clinically and by TVS.

$40 \%$ of patients in Group B i.e. the vaginal group required $400 \mu \mathrm{g}$ Misoprostol sublingually which is 80 out of 200 in the arm. Whereas $28.5 \%$ in Group A i.e. the sublingual group required a top up dosage which is 57 out of 200 .

Significantly higher number of patients had a quicker abortion in sublingual route at the end of D7 (97.50\% vs. $88.50 \%$ ) as compared to vaginal route. 2 patients required $\mathrm{S} \& \mathrm{E}$ in Group B whereas all responded successfully in Group A. Second sublingual dose of Misoprostol $400 \mu \mathrm{g}$ was required by more patients at the end of D7 as assessed both by TVS and gynaecological findings.

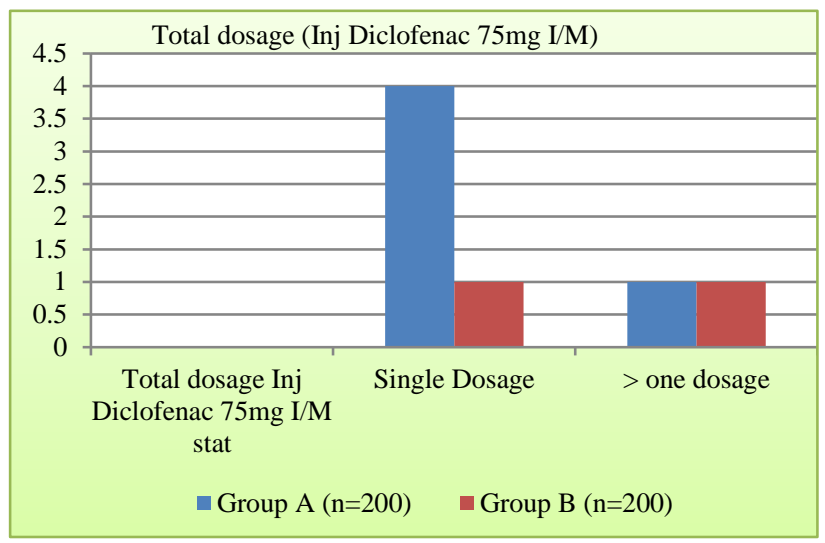

Figure 3: Requirement of parenteral analgesics (non opioid).

4 patients required Parenteral analgesic in Group A possibly because of quicker and more rapid initiation of powerful uterine contractions in a short span of time within hours of administration as compared to slower and gradual absorption by vaginal route. Second dosage was requested by equal number of patients in both arms on administration of second sublingual dose of misoprostol, possibly because of lower pain threshold.

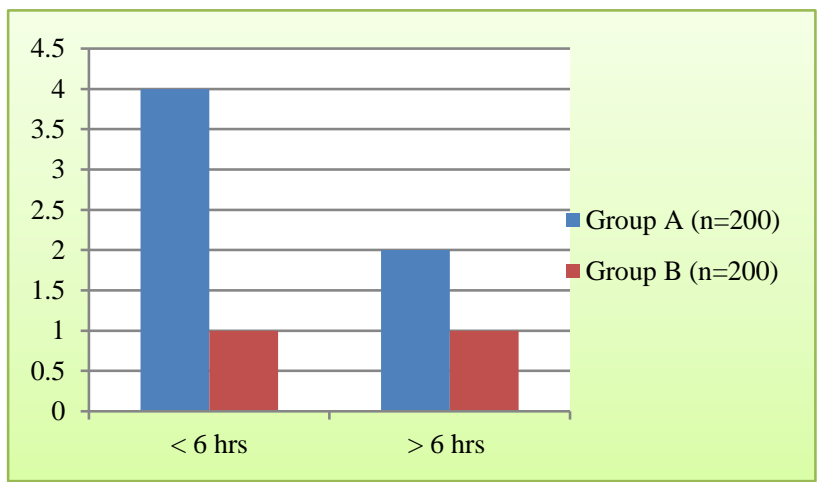

Figure 4: Requirement of hospitalization.

Figure 3 and 4 are comparable and those who required Parenteral analgesia were also detained briefly in the hospital for observation, reassurance and psychological support.

2 patients required surgical evacuation in Group B, one on D15 on request on OPD basis by MVA and second on D56 due to continuous bleeding vaginally and retained 
products (RPOC) on Ultrasound (TVS). Products were sent for Histopathology (HPE) for exclusion of Gestational Trophoblastic Disease (GTN), microscopic examination confirmed RPOC.

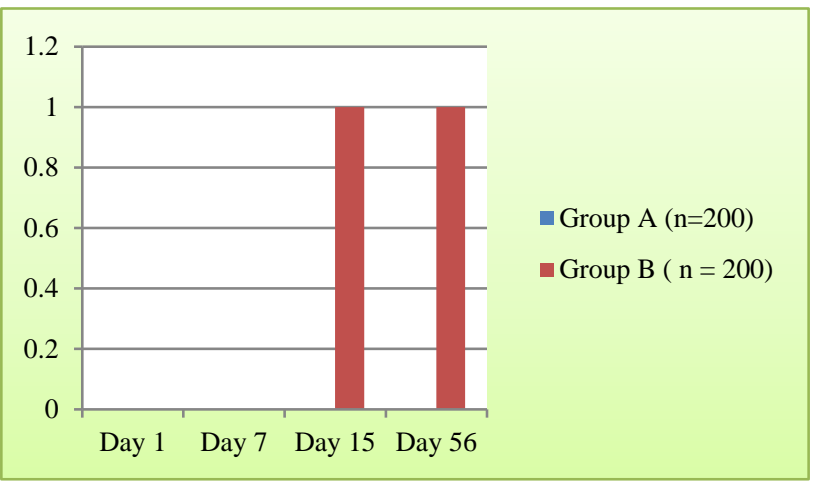

Figure 5: Patients requiring S\&E for completion of abortion.

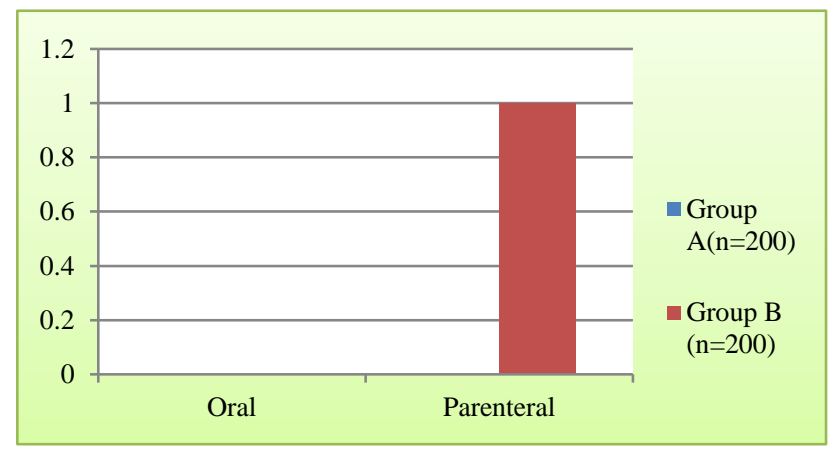

Figure 6: Requirement of antibiotics.

None required oral antibiotics in either arm and 2 patients in Group B who underwent surgical evacuation were administered single dose of prophylactic antibiotic as per institution protocol. Further requirement of oral or Parenteral antibiotic was not necessary in either arm.

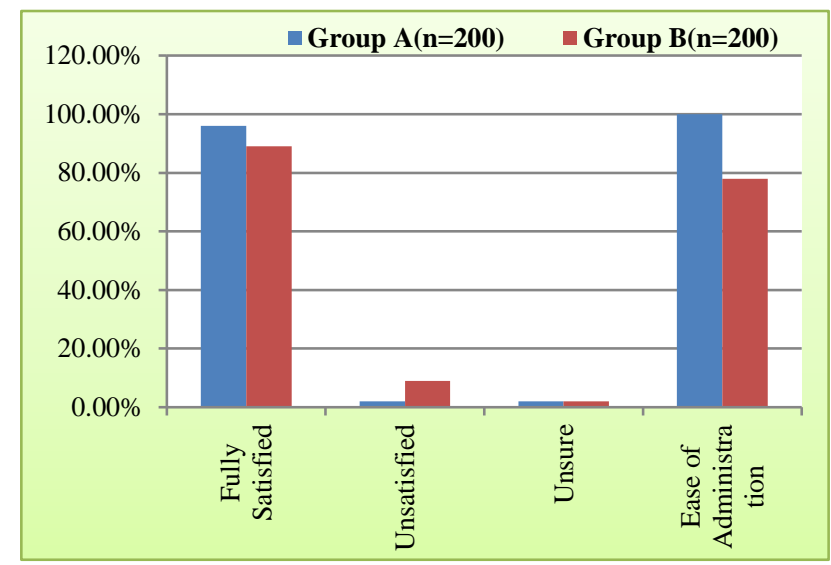

Figure 7: Satisfaction levels based on questionnaire.

As can be seen most patients were fully satisfied as it was self administered and physician monitored. Most had anxiety regarding completion of procedure as they had knowledge only of conventional methods of termination and had minimal knowledge or access to easy self administered drugs. Tendency to misuse and refusal of permanent method of contraception was also higher as compared to those undergoing surgical evacuation under anaesthesia. Group A patients were most comfortable regarding ease of administration and Group B patients had issues regarding hygiene and privacy, as they were already bleeding after administration of Mifepristone and unabsorbed tablets escaped from vagina spontaneously leading apprehension. They faced difficulty in self placement in posterior fornix and often returned to hospital for assistance. Lack of sterile gloves was also an important issue. In our current study it's observed that women with Intrauterine pregnancy of 9 weeks or less can safely self administer $200 \mathrm{mg}$ Mifepristone followed by Misoprostol sublingually after 48 hours, which we found was more acceptable route as some young women are reluctant to administer the dosage vaginally due to privacy and hygiene issues. Majority of patients had complete medical abortion following both methods of termination and comparable side effects. However sublingual route had faster action and better patient satisfaction.

\section{DISCUSSION}

The World Health Organization advocates use of lower dosage of Mifepristone as early as $1991^{(3)}$. Extensive medical research and subsequent field trials have proven the efficacy of lower dosage with various routes of administration of Misoprostol with additional benefits, tolerability and cost effectiveness ${ }^{(4)}$. Mifepristone is the first steroidal, antiglucocorticoid, antiprogesterone; acting at the receptor level to be developed. It has rapid absorption and a long half life of 25 to 30 hours. The serum transport protein $\alpha 1$ and glucoprotein (AAG) regulates serum kinetics and its binding to AAG limits bioavailability; explaining low metabolic clearance rate of $0.55 \mathrm{l} / \mathrm{kg} /$ day and low volume distribution. Similar serum concentrations of Mifepristone exceeding 100mg can be explained by saturation of binding capacity of AAG.

The drug also undergoes hepatic first pass metabolism; therefore inspite of $70 \%$ absorption via the oral route; bioavailability is only $40 \%$. Recommended dosage of Mifepristone varies from $50 \mathrm{mg}$ to $200 \mathrm{mg}$ orally for cervical priming. Earlier dosage used was $600 \mathrm{mg}$ with no obvious clinical advantage. The drug has now been approved for preinduction cervical ripening for TOLAC cases and studies are underway. Misoprostol concentration in blood reaches its peak 12 minutes after oral administration. Tang et al compared the pharmacokinetics of different routes of misoprostol administration, namely sublingual, oral and vaginal by measuring its acid metabolite using gas chromatography/ tandem mass spectrometry. ${ }^{5}$ Sublingual misoprostol achieved highest peak serum concentration and the area 
under the curve was also significantly higher than other groups, suggesting that this may be the most potent route of administration of the drug. The abortion took mean of 14 hours to finish and bleeding took 12 days on an average to stop. Mifepristone and misoprostol in combination cause excellent cervical ripening, uterine smooth muscle contractions and dilatation of cervix. Absorption via the vaginal route is slow and erratic and maximum levels are seen in serum at approximately 80 minutes and falls slowly; therefore has longer duration of action and longer bioavailability than oral route. ${ }^{6}$

Mucous membrane of oral cavity has a very rich blood supply and a potentially faster and more reliable drug delivery path which directly acts on uterus and has higher bioavailability and also bypasses the liver where the drug is metabolized. Misoprostol when administered sublingually is completely absorbed within 20 minutes, reaches maximum serum levels in 26 minutes, initiating powerful uterine contractions in 11.5 minutes and peaking in 52 minutes with better bioavailability than oral route and similar to vaginal route. ${ }^{7}$

Misoprostol is most commonly manufactured as $200 \mu \mathrm{g}$ tablet intended for oral administration although varying strengths of the drug are available for different purpose and usage. ${ }^{8}$ It's stable at ambient temperature while other products require refrigeration or freezing. ${ }^{9}$ Some are available as parenteral preparation only. It's inexpensive and easily available. ${ }^{10}$ Successful use of misoprostol implies complete evacuation of the uterus without recourse to surgical intervention. Infrequently, surgical intervention may be required due to heavy bleeding or on request of the patient. Average efficacy based on any route of administration was approximately $95 \%$ and success rate as high as $99 \%$. Completeness of abortion was assessed based on clinical symptoms and signs, internal examination of with a 6 weeks uterine size and a trilaminar endometrium with Endometrial thickness less than $14 \mathrm{~mm}$ (index for the current study). Misoprostol provides effective, safe, acceptable outpatient option for abortion for women who donot have access to surgical methods or for those wish to avoid invasive in patient procedures.

It has no long term effects on women's health and serious side effects are nonexistent. Surgical evacuation procedures include Dilatation and Currettage (D\&C), Electric Vacuum Aspiration (EVA), Manual Vacuum Aspiration (MVA). These methods have high success rate but carry a risk of perforation, cervical laceration and infections. Misoprostol obviates the need for surgery, anaesthesia, referral to higher centres and can be integrated into existing settings in resource poor nations with minimal post abortion care (PAC) facilities. ${ }^{11}$ High efficacy rates with acceptable side effects profiles have been obtained with both a single $600 \mu \mathrm{g}$ oral dose and with a single $400 \mu \mathrm{g}$ sublingual dose of misoprostol. Both regimens work equally well. Repeated dosing within a short interval does not seem to improve efficacy. ${ }^{12-17}$

Table 2: Common expected side effects and their management are as enumerated in the table.

\begin{tabular}{|lll|}
\hline Symptom & Description & Management \\
\hline Pain/ Cramping & Classically described as severe dysmenorrhoea & Analgesics, hot water bag, Drotavarine \\
\hline $\begin{array}{l}\text { Diaphoresis/ } \\
\text { Anxiety/Palpitations }\end{array}$ & Transient $<24 \mathrm{hrs}$ & Antipyretics, Report if $>24$ hrs \\
\hline Heavy bleeding & Passage of clots without postural hypotension & $\begin{array}{l}\text { Analgesics, observation } \\
\text { haemostatic agents }\end{array}$ \\
\hline Nausea/vomiting & Transient $<6 \mathrm{hrs}$ & Antiemetics \\
\hline Diarrhoea & Transient $<24 \mathrm{hrs}$ & Reassure, ORS \\
\hline Infection & Rare & Document, oral antibiotics \\
\hline $\begin{array}{l}\text { Dizziness/ } \\
\text { Syncope }\end{array}$ & Postural & Comfort fluids, lying, sitting position \\
\hline RPOC & Continuous pain and bleeding/spotting PV & Repeat Misoprostol, offer S \& E \\
\hline Urinary symptoms & Polyuria, dysuria & Bladder relaxants, drotavarine, Tolteridine \\
\hline Drug Rash & Transient, pruritic, Blotchy & $\begin{array}{l}\text { Antihistaminic (H1 \& H2 receptor } \\
\text { blockers), emollients }\end{array}$ \\
\hline Mastalgia & Transient $<24 \mathrm{hrs}$ & Loose clothing, Mild diuretics \\
\hline
\end{tabular}

There is no clinical reason to withhold Medical MTP in women with previous Caesarean section. It also works with equal efficiency in women who require services for Early Pregnancy Failure or those who have induced abortions with misoprostol unsuccessfully. ${ }^{18}$ The dosage can be physician administered or self administered under guidance of a Health Care Worker. Ultrasound (TVS) although not mandatory is desirable for exclusion of Extrauterine Gestation; however the biggest drawback is over interpretation of normal amounts of debris in the 
uterus, leading to unnecessary surgical intervention. Providers should be aware that women treated successfully with misoprostol have been found to have a wide range of endometrial thickness on TVS follow up; therefore it is recommended that the decision to perform surgical evacuation be based on clinical signs rather than imaging. ${ }^{19}$ All women should be informed that fertility returns quickly following an abortion and should be given appropriate contraceptive advice.

Lactating mothers are advised to discard breast milk few hours after Misoprostol administration. There is no reason to observe the women in the hospital and self administration is known to be safe and has same efficacy with low rates of complications. Excessive bleeding warranting transfusion or infection requiring antibiotics is remote and is as rare as in surgical evacuation. However all women should have access to emergency facility. Acceptance of contraception management was easier and patients were easily convinced for temporary methods of contraception.

\section{Uncommon clinical and laboratory findings}

Hypotension, Bradycardia, Raised ESR, Thrombocytopenia, Glycosuria, Hyperuricemia, raised creatinine, Haematuria, Increased Serum Alkaline Phosphatase, deranged Liver functions, raised amylase, unspecified arrhythmias, Anaemia, lowers Intra ocular pressure. TVS may show fluid in POD upto 56 to 60 days in upto $40 \%$ of patients in post abortal status not requiring any treatment except reassurance. There's no evidence of infection or Sepsis and collection in POD resolves spontaneously in $100 \%$ patients. In conclusion to terminate early pregnancy by medical methods ; sublingual administration of misoprostol ranging from $400 \mu \mathrm{g}$ to $600 \mu \mathrm{g}$ after oral administration of mifepristone or sole administration of sublingual misoprostol are effective therapeutic methods. In this study 200mg mifepristone followed by $600 \mu \mathrm{g}$ misoprostol resulted in complete abortion in $100 \%$ cases with only few requiring second dose of misoprostol. Since sublingual administration of misoprostol bypasses the hepatic first pass its efficacy is theoretically better than that of oral route.

\section{CONCLUSION}

The most widely used dosage for first trimester abortion involves oral administration of $200 \mathrm{mg}$ of mifepristone followed by $600 \mu \mathrm{g}$ misoprostol orally or vaginally 48 hours later. There is a need for safe, fast, efficient, acceptable and patient friendly method of MTP. We compared Sublingual and Vaginal routes of administration of Misoprostol after cervical priming with Mifepristone and found sublingual method better and more acceptable. We recommend this protocol as a safe outpatient method for termination in early pregnancy of less than 9 weeks Period of Gestation.

\section{Limitations of this study}

Number of patients in this study group was small. Larger study groups with RCT and more stastical tests may be applied for reliable study outcome. Long term follow up of patients is required.

Funding: No funding sources Conflict of interest: None declared

Ethical approval: The study was approved by the Institutional Ethics Committee

\section{REFERENCES}

1. Nothnagle M, Taylor JS. Medical methods for first trimester abortion. Am Fam Physician. 2004;70:813.

2. Hamoda H, Flett GM. Medical termination of pregnancy in the early first trimester. J Fam Plann Reprod Health Care. 2005;31:10-4.

3. World Health Organization. Pregnancy termination with mifepristone and gemeprost: a multicenter comparison between repeated doses and a single dose of mifepristone. Fertil Steril. 1991;56:32-40.

4. Newhall EP, Winikoff B. Abortion with mifepristone and misoprostol: regimens, efficacy, acceptability and future directions. Am J Obstet Gynecol. 2000;183:S44-53.

5. Tang OS, Schweer H, Seyberth HW, Lee SWH, Ho PC. Pharmacokinetics of different routes of administration of misoprostol. Hum Reprod. 2002;17:332-6.

6. Zieman M, Fong SK, Benowitz NL, Banskter D, Darney PD. Absorption kinetics of misoprostol with oral or vaginal administration. Obstet Gynecol. 1997;90:88-92.

7. Aronsson A, Bygdeman M, Gemzell-Danielsson K. Effect of misoprostol on uterine contractility following different routes of administration. Hum Reprod. 2004;19:81-4.

8. Goldberg AB, Greenberg M, Darney PD. Misoprostol and pregnancy. $\mathrm{N}$ Engl $\mathrm{J}$ Med. 2001;344(1):38-47.

9. Shannon CS, Winikoff B, eds. Misoprostol: An emerging technology for women's health. Report of a Seminar: May 7-8, 2001. New York Population Council, 2004.

10. Shannon C. Misoprostol: Investigator's Brochure. Gynuity health Projects, New York; 2006.

11. Sahin HG, Sahin HA, Kocer M. Randomized outpatient clinical trial of medical evacuation and surgical curettage in incomplete miscarriage. Eur $\mathbf{J}$ Contracept Reproductive Health Care. 2001;6(3):141-4.

12. Shwekerela B, Kalumuna R, Kipingili R, Mashaka $\mathrm{N}$, Westheimer E, Clark W, et al. Misoprostol for treatment of incomplete abortion at the regional hospital level: Results from Tanzania. BJOG 2007;114(11):1363-7. 
13. Diop A, Raghavan S, Rakotovao JP, Comendant R, Blumenthal PD, Winikoff B. Comparison of two routes of administration for misoprostol in the treatment of incomplete abortion: A randomised control trial. Contraception. 2009;79:456-62.

14. Bique C, Ustá M, Debora B, Chong E, Westheimer E, Winikoff B. Comparison of Misoprostol and manual vacuum aspiration for the treatment of incomplete abortion. Int $\mathbf{J}$ Gynecol Obstet. 2007;98(3):222-6.

15. Dao B1, Blum J, Thieba B, Raghavan S, Ouedraego M, Lankoande $\mathbf{J}$, et al. Is misoprostol a safe, effective, acceptable alternative to manual vacuum aspiration for post abortion care? Results from a randomised control trial in Burkina Faso, West Africa. BJOG. 2007;114(11):1368-75.

16. Weeks A, Alia G, Blum J, Winikoff B, Ekwaru P, Durocher $\mathrm{J}$, et al. A randomised trial of oral misoprostol versus manual vacuum aspiration for the treatment of incomplete abortion in Kampala, Uganda. Obstet Gynecol. 2005;106(3):540-7.
17. Blanchard K, Taneepanichskul S, Kiriwat O, Sirimai $\mathrm{K}$, Svirirojana N, Mavimbela N, et al. Two regimens of misoprostol for treatment of incomplete abortion. Obstet Gynecol. 2004;103:860-5.

18. Nguyen TN, Blum J, Durocher J, Quan TT, Winikoff B. A randomised controlled study comparing $600 \mu \mathrm{g}$ versus $1200 \mu \mathrm{g}$ oral misoprostol for medical management of incomplete abortion. Contraception. 2005;72(6):438-42.

19. Reeves MF, Fox MC, Lohr PA, Creinin MD. Endometrial thickness following medical abortion is not predictive of subsequent surgical intervention. Ultrasound Obstet Gynecol. 2009;34(1):104-9.

Cite this article as: Devi LT, Nimonkar R. Comparative evaluation of two commonly administered regimens of mifepristone and misoprostol for first trimester abortion. Int J Reprod Contracept Obstet Gynecol 2018;7:xxx-xx. 